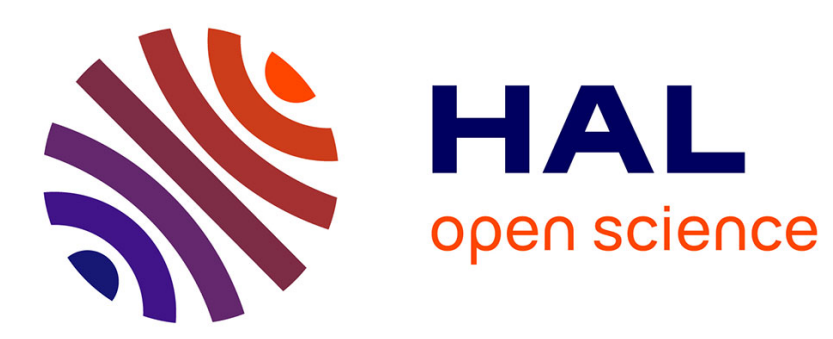

\title{
Modification de revêtements de MoSi2 sur molybdène par le germanium
}

\author{
P. Steinmetz, J. Steinmetz, B. Roques
}

\section{To cite this version:}

P. Steinmetz, J. Steinmetz, B. Roques. Modification de revêtements de MoSi2 sur molybdène par le germanium. Journal de Physique IV Proceedings, 1993, 03 (C9), pp.C9-909-C9-919. 10.1051/jp4:1993994 . jpa-00252436

\section{HAL Id: jpa-00252436 https://hal.science/jpa-00252436}

Submitted on 1 Jan 1993

HAL is a multi-disciplinary open access archive for the deposit and dissemination of scientific research documents, whether they are published or not. The documents may come from teaching and research institutions in France or abroad, or from public or private research centers.
L'archive ouverte pluridisciplinaire HAL, est destinée au dépôt et à la diffusion de documents scientifiques de niveau recherche, publiés ou non, émanant des établissements d'enseignement et de recherche français ou étrangers, des laboratoires publics ou privés. 


\title{
Modification de revêtements de $\mathrm{MoSi}_{2}$ sur molybdène par le germanium
}

\author{
P. Steinmetz, J. Steinmetz et B. Roques
}

Laboratoire de Chimie du Solide Minéral, Faculté des Sciences de l'Université de Nancy I, B.P. 239, 54506 Vandoeuvre Cedex

\begin{abstract}
Résumé. - La protection du molybdène contre l'oxydation haute température peut être réalisée par simple siliciuration. Ce procédé conduit à la formation de couches superficielles de disiliciure $\mathrm{MoSi}_{2}$, qui confèrent au métal une bonne résistance en conditions isothermes. Les principales limitations de ce procédé tiennent à la sensibilité au cyclage thermique de la couche de silice qui se forme sur $\mathrm{MoSi}_{2}$, et à l'interdiffusion entre ce composé et le molybdène qui conduit à la formation du composé $\mathrm{Mo}_{5} \mathrm{Si}_{3}$, très oxydable. Lincorporation de germanium au disiliciure peut apporter de ces deux points de vue une amélioration sensible. Elle se traduit en effet par une augmentation de la résistance du film de silice aux chocs thermiques. Elle présente en outre l'intérêt de pouvoir être réalisée facilement par cémentation du molybdène avec des céments binaires SiGe. Létude de la diffusion dans le système $\mathrm{Mo} / \mathrm{Mo}(\mathrm{Si}, \mathrm{Ge})_{2}$ montre par ailleurs que l'incorporation de germanium au disiliciure modifie de façon sensible les processus et la vitesse de croissance de la couche intermédiaire de $\mathrm{Mo}_{5} \mathrm{Si}_{3}$.
\end{abstract}

\begin{abstract}
Molydenum disilicide coatings can afford a good protection against H.T. oxidation to molybdenum substrates. Their main use limitation is their sensitivity to thermal cycling, due to the mismatch between expansion coefficients of silica and the intermetallic substrate and to interdiffusion between coating and molybdenum substrate which leads to $\mathrm{Mo}_{5} \mathrm{Si}_{3}$ formation, a compound very sensitive to H.T. oxidation. Alloying of $\mathrm{MoSi}_{2}$ with germanium can have a beneficial influence from these two points of view. Germanium increases the resistance of the silica protective film to thermal cycling; it also lowers the diffusion rate in the $\mathrm{MoSi}_{2} / \mathrm{Mo}$ system.
\end{abstract}

\section{Introduction.}

La siliciuration superficielle du molybdène est une méthode intéressante de protection du molybdène et de ses alliages contre l'oxydation à chaud. En effet, le traitement est facile à réaliser et les revêtements du disiliciure $\mathrm{MoSi}_{2}$ sont passivés dans un large domaine de température et de pression d'oxygène, grâce à la formation d'un film de silice.

Si l'on excepte les risques d'accidents mécaniques provoquant la fissuration ou le décollement des revêtements, ce système de protection présente toutefois deux inconvénients :

- il est thermodynamiquement instable et évolue par diffusion à l'état solide avec formation des composés intermédiaires $\mathrm{Mo}_{5} \mathrm{Si}_{3}$ et $\mathrm{Mo}_{3} \mathrm{Si}$ qui sont beaucoup plus oxydables que le disiliciure ; l'interaction du revêtement et du substrat devient assez rapide aux températures élevées et limite donc la durée de la protection ;

- il résiste moins bien aux cycles thermiques qu'aux chauffages isothermes, probablement 
à cause de la différence entre les coefficients de dilatation du disiliciure et de la silice.

C'est en vue d'atténuer ces défauts que nous avons étudié la réalisation de revêtements de disiliciure de molybdène allié au germanium. Les travaux de Fitzer et al. $[1,2,4]$ et de Schlichting [3] ont en effet montré que l'addition de dioxyde de germanium à la silice permet d'ajuster les dilatations thermiques du revêtement et de sa couche protectrice et se traduit par une nette amélioration de sa tenue en cycles thermiques. Nous avons ainsi étudié la cémentation du métal par des alliages Si-Ge, puis l'effet du germanium sur la diffusion dans les pièces revêtues.

\section{Procédure expérimentale.}

2.1 CÉMENTATIONS. - Les céments ont été préparés à partir de mélanges stoechiométriques des poudres élémentaires ; un traitement de 48 heures à $1100^{\circ} \mathrm{C}$ en ampoule de quartz en présence de tétrachlorure de silicium $\left(10^{4}\right.$ Pascal à $\left.20^{\circ} \mathrm{C}\right)$, produit des alliages homogènes dont la composition est vérifiée par une mesure de paramètre cristallin.

Les échantillons métalliques étaient ensuite traités en présence de ce cément, dans des ampoules de quartz qui étaient scellées après avoir été soumises aux deux opérations suivantes :

- dégazage sous des pressions inférieures à 100 Pascal et à des températures croissantes jusqu'à $300^{\circ} \mathrm{C}$;

- après refroidissement à la température ambiante, introduction de tétrachlorure de silicium sous une pression de $10^{4}$ Pascal.

2.2 RECUITS DE DIFFUSION. - Les interactions entre le molybdène et les revêtements réalisés par cémentation ont été étudiées par des recuits sous argon de pièces revêtues, à des températures comprises entre 1000 et $1500^{\circ} \mathrm{C}$.

\section{Résultats.}

3.1 CÉmentation du molybdène PAR DeS alliages Silicium-Germanium. - Les essais de cémentation réalisés avec des alliages $\mathrm{Si}-\mathrm{Ge}$ de composition variable $\left(\mathrm{Si}_{1-x} \mathrm{Ge}_{x}\right.$, avec $0<x<0,5$ ), conduisent à la croissance sur le molybdène, d'une couche à cristallisation basaltique, constituée de la phase $\operatorname{Mo}\left(\mathrm{Si}_{1-y} \mathrm{Ge}_{y}\right)_{2}$, de type $\mathrm{G}_{11 \mathrm{~b}}$ (Fig. 1).

La teneur en germanium varie toujours de la même manière suivant la pénétration (Fig. 2) :

- en surface, y est sensiblement nul ;

- le germanium ne commence à apparaître qu'à partir d'une épaisseur d'autant plus grande que le cément est plus pauvre en germanium ; sa concentration augmente ensuite progressivement jusqu'à l'interface avec le métal.

Quand les céments sont très riches en silicium $(x=0,01$ à 0,2$)$ les couches sont uniformes et leur teneur maximale en germanium, $y_{\mathrm{m}}$, ne dépend que des valeurs de $x$ et de la température de traitement.

Les céments plus riches en germanium $(x=0,3$ à 0,45$)$ conduisent à des revêtements dont l'épaisseur et $y_{\mathrm{m}}$ sont moins bien définis, particulièrement à $1100^{\circ} \mathrm{C}$. A la surface d'un même échantillon, ces deux caractères fluctuent corrélativement ; $y_{\mathrm{m}}$ crôtt ou décroît comme l'épaisseur. Si $x$ et la température sont fixés, les valeurs extrêmes de $y_{\mathrm{m}}$ sont tout de même sensiblement indépendantes de la durée de cémentation. 


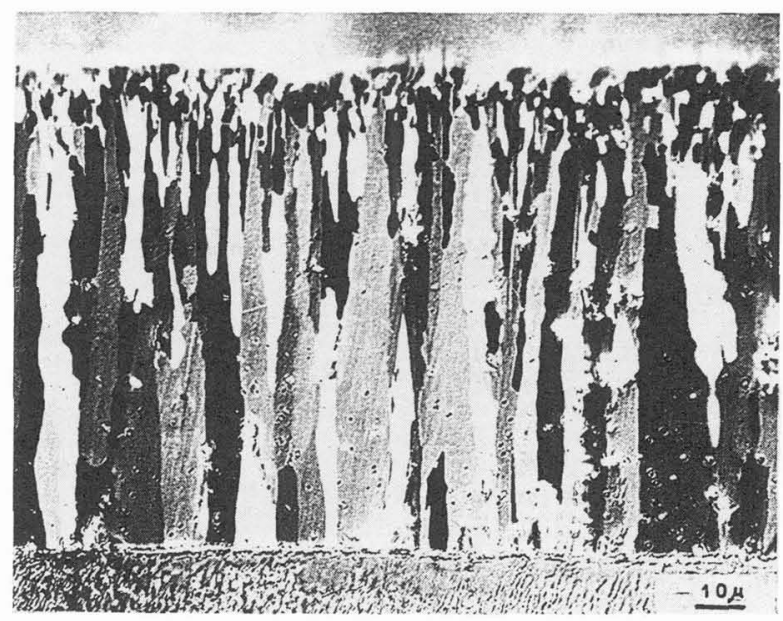

Fig. 1. - Mo cémenté par $\mathrm{Si}_{0,8} \mathrm{Ge}_{0,2} 10 \mathrm{~h} 15 \mathrm{mn}$ à $1100^{\circ} \mathrm{C}$.

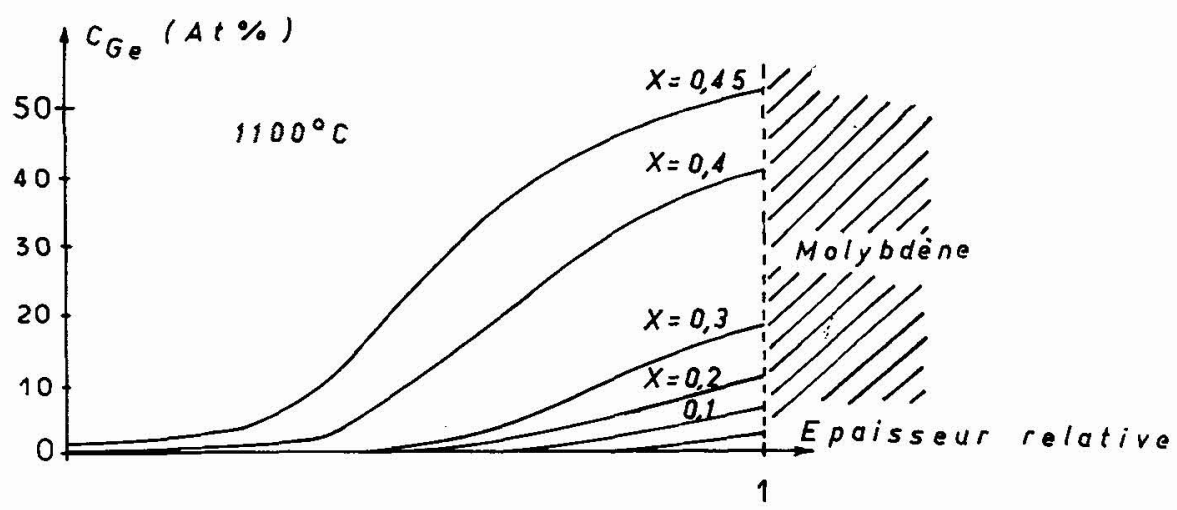

Fig. 2. - Répartition du germanium en fonction de la composition du cément.

La valeur de $y_{\mathrm{m}}$ augmente en fonction de $x$, assez lentement à $1000^{\circ} \mathrm{C}$ et beaucoup plus vite à $1100^{\circ} \mathrm{C}$, au moins pour $x>0,3$ (Fig. 3); par ailleurs, $y_{\mathrm{m}}$ n'est supérieure à $x$ que dans les conditions suivantes : $x>0,3$ et température égale à $1100^{\circ} \mathrm{C}$.

En dehors de ces conditions, le rapport des teneurs en germanium et silicium est plus faible dans le revêtement que dans le cément ; ce dernier s'enrichit donc progressivement en germanium. Cet écart peut être dû à des conditions de transport en phase gazeuse différentes pour le silicium et le germanium.

Aux deux températures étudiées, l'épaisseur des revêtements croît avec la durée du traitement suivant une loi parabolique : $e=K t^{1 / 2}$.

A $1000{ }^{\circ} \mathrm{C}$, la constante de vitesse $K$ est sensiblement indépendante de la teneur en germanium du cément : à $1100^{\circ} \mathrm{C}$, elle augmente avec $x$, très rapidement au delà de $x=0,3$ (Fig. 4). 


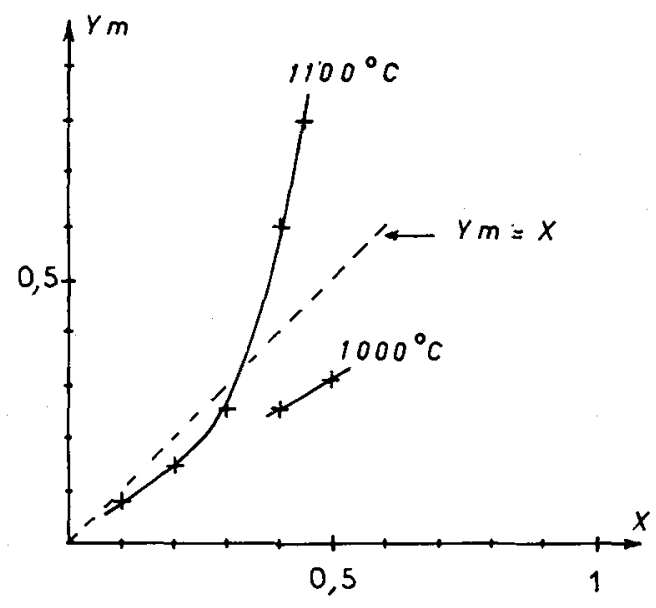

Fig. 3. - Evolution de la teneur en germanium à l'interface molybdène/revêtement, en fonction de la composition du cément.

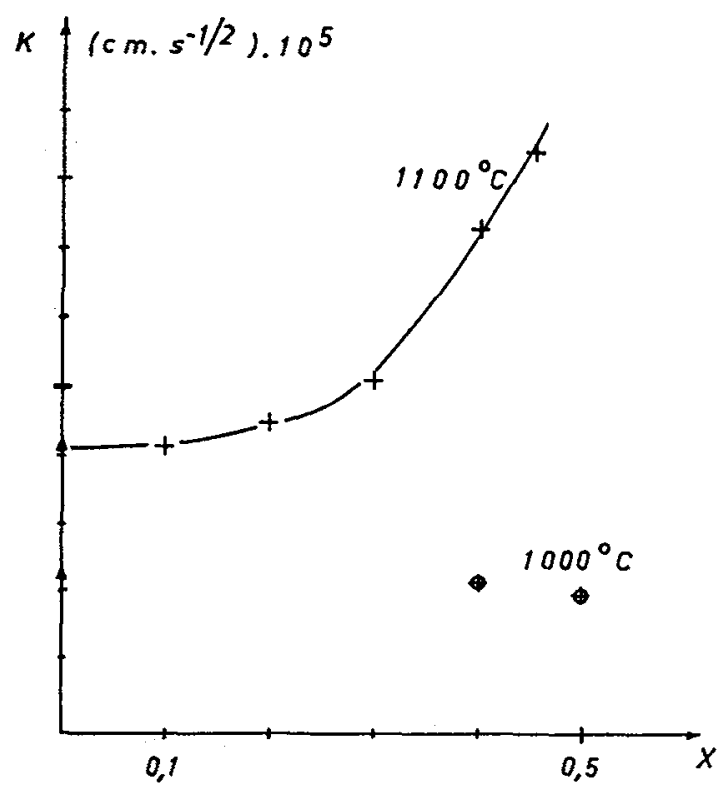

Fig. 4. - Evolution de la constante de vitesse pour la croissance du revêtement, en fonction de la teneur en germanium du cément.

3.2 RECUITS DE DIFFUSION. - Les échantillons étudiés ont été préalablement cémentés avec l'alliage $\mathrm{Si}_{0,8} \mathrm{Ge}_{0,2}$, pendant $10 \mathrm{~h} 15 \mathrm{mn}$ à $1100^{\circ} \mathrm{C}$, en présence de $\mathrm{SiCl}_{4}$. Ces conditions conduisent à l'obtention d'un revêtement très régulier et assez épais (100 $\mu \mathrm{m})$ de disiliciure faiblement allié au germanium. La distribution de cet élément est caratérisée par une teneur maximale à l'interface avec le métal $\left(y_{\mathrm{m}}=0,15\right)$ et un appauvrissement progressif vers la 
surface, avec disparition quasi-totale à mi-épaisseur.

Des recuits sous argon, de durées variables, ont ensuite été effectués à des températures comprises entre 1000 et $1500^{\circ} \mathrm{C}$. Ils conduisent d'une part à une redistribution du germanium dans la couche superficielle et d'autre part à la formation d'une couche intermédiaire.

La redistribution du germanium dans la couche superficielle conduit à une uniformisation de la teneur de cet élément $(2,5$ at\%), d'autant plus rapide que la température est élevée : elle demande $16 \mathrm{~h}$ à $1100^{\circ} \mathrm{C}$ et seulement quelques minutes à $1400^{\circ} \mathrm{C}$.

La composition de la couche de diffusion peut être exprimée par la formule $\mathrm{Mo}_{5}\left(\mathrm{Si}_{1-z} \mathrm{Ge}_{z}\right)_{3}$ mais sa teneur en germanium ne varie pas de façon continue avec la pénétration. Il y a en effet trois zones qui se distinguent à la fois par leur cristallisation et leur teneur en germanium (Fig. 5).

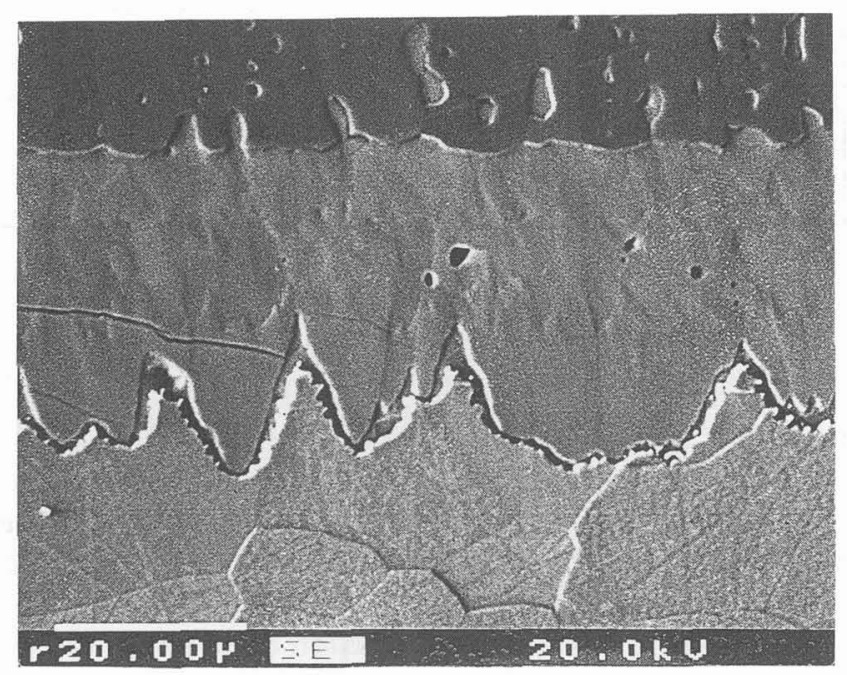

Fig. 5. - $\mathrm{Mo} / \mathrm{Mo}(\mathrm{Si}, \mathrm{Ge})_{2}$, recuit $144 \mathrm{~h}$ à $1200^{\circ} \mathrm{C}$ sous argon.

Quand la diffusion a atteint un régime stationnaire, une fois que les effets de l'homogénéisation initiale de la couche superficielle se sont effacés, ces différentes zones ont les caractéristiques suivantes (Fig. 6) :

- la première (I) adjacente au revêtement de $\mathrm{Mo}(\mathrm{Si}, \mathrm{Ge})_{2}$ et d'épaisseur $e_{\mathrm{I}}$, a une teneur en germanium très faible $(\sim$ lat\%), sensiblement uniforme et indépendante de la durée et de la température du recuit ;

- la zone intermédiaire (II.1), d'épaisseur $e_{\text {II.1, }}$, présente un fort gradient de composition avec une teneur maximale en germanium au voisinage de la zone interne ; cette teneur est sensiblement indépendante de la durée d'un recuit isotherme et elle croît en fonction de la température : de 1200 à $1500^{\circ} \mathrm{C}$ elle passe de 3 à 5 at\% environ ;

- enfin, la zone adjacente au molybdène (II.2), d'épaisseur $e_{\mathrm{II} .2}$, ne contient pas de germanium.

On remarque que le rapport $\frac{e_{\mathrm{I}}}{e_{\mathrm{I}}+e_{\mathrm{II}}}$ conserve une valeur voisine de $3 / 7$ quelles que soient la température et la durée de la diffusion en régime stationnaire. Cette valeur est identique 

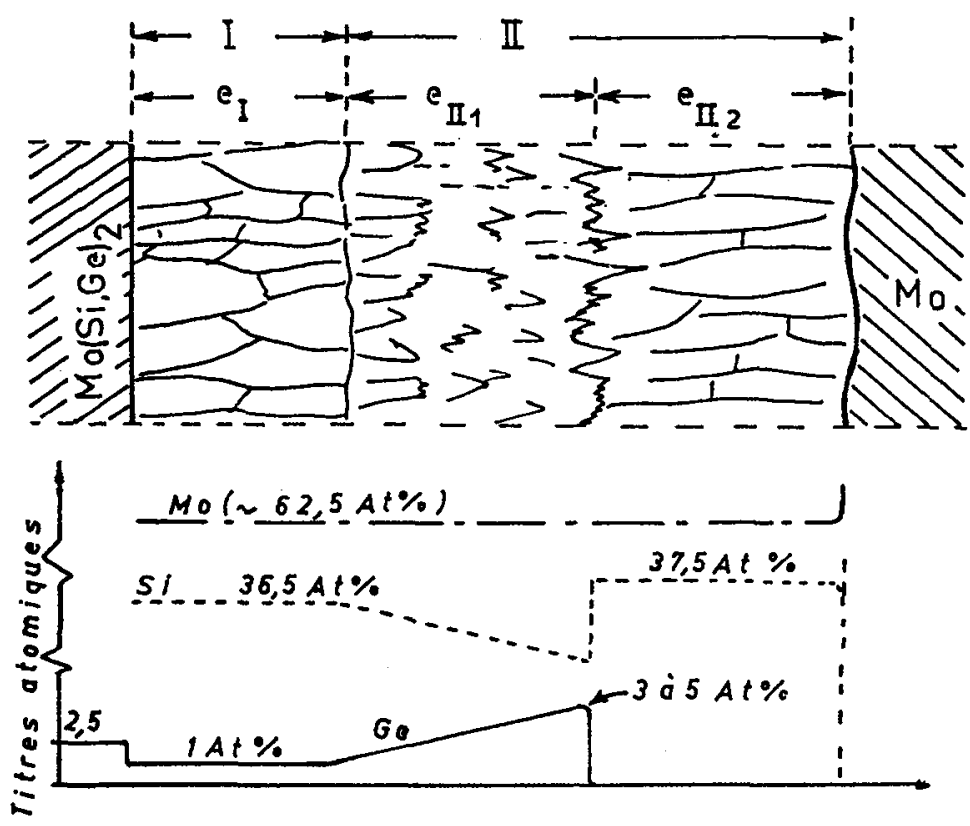

Fig. 6. - Diffusion dans le système $\mathrm{Mo} / \mathrm{Mo}(\mathrm{Si}, \mathrm{Ge})_{2}$.

à celle qui a été obtenue par ailleurs pour la croissance de $\mathrm{Mo}_{5} \mathrm{Si}_{3}$ dans le couple $\mathrm{Mo}-\mathrm{MoSi} \mathrm{i}_{2}$ [5-13] et indique une diffusion préférentielle du silicium et du germanium.

A température constante, l'épaisseur totale $\left(e_{\mathrm{I}}+e_{\mathrm{II}}\right)$ de la couche intermédiaire de $\mathrm{Mo}_{5}\left(\mathrm{Si}_{1-z} \mathrm{Ge}_{z}\right)_{3}$ varie linéairement avec la racine carrée de la durée des essais : $e=k \cdot \sqrt{t}$ (Fig. 7).

Les variations de la constante de vitesse en fonction de la température obéissent sensiblement à une loi d'Arrhenius qui s'exprime par :

$$
k\left(\mathrm{~cm} \cdot \mathrm{s}^{-1 / 2}\right)=1,58 \cdot \exp \frac{-36400}{R T}
$$

Aux températures inférieures à $1600{ }^{\circ} \mathrm{G}$, la croissance du composé ternaire $\mathrm{Mo}_{5}$ $\left(\mathrm{Si}_{1-z} \mathrm{Ge}_{z}\right)_{3}$ est moins rapide que celle du siliciure $\mathrm{Mo}_{5} \mathrm{Si}_{3}$ dans le couple $\mathrm{Mo}-\mathrm{MoSi}_{2}$ (Fig. 8) $[11,13]$.

L'exploitation de ces résultats est compliquée du fait de la répartition inégale du germanium dans le revêtement des échantillons étudiés ; les conditions de diffusion évoluent tant que la composition de ces revêtements ne s'est pas uniformisée.

Pour éviter cette difficulté, on peut utiliser la technique des couples de diffusion en recuisant sous argon, après collage, deux plaquettes de molybdène dont l'une comporte un revêtement épais de l'alliage $\mathrm{Mo}\left(\mathrm{Si}_{1-y} \mathrm{Ge}_{y}\right)_{2}$, homogénéisé préalablement par recuit.

Cette méthode se prête assez mal à l'étude de l'évolution des processus de diffusion avec la teneur en germanium de l'alliage $\mathrm{Mo}\left(\mathrm{Si}_{1-y} \mathrm{Ge}_{y}\right)_{2}$; nous lui avons donc préféré la cémentation du molybdène par des alliages homogènes $\mathrm{Mo}\left(\mathrm{Si}_{1-y} \mathrm{Ge}_{y}\right)_{2}$.

Ces traitements assurent en effet un régime de diffusion stationnaire, au moins dans les conditions opératoires adoptées :

- cémentation en système fermé et isotherme ; 


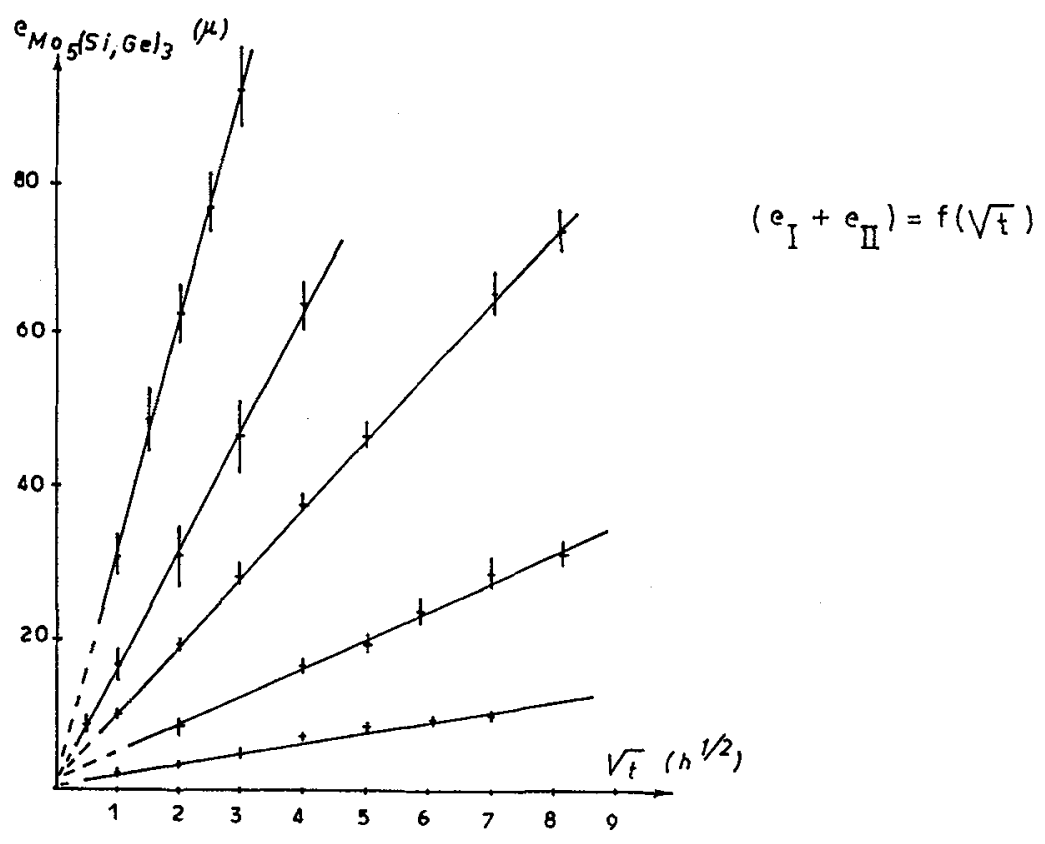

Fig. 7. $-\left(e_{\mathrm{I}}+e_{\mathrm{II}}\right)=f(\sqrt{t})$.

- contrôle de la composition du cément et de la pression (ou quantité) d'activant introduite dans le système.

Toutefois, l'examen des pièces traitées ne montre que les couches (II) formées à partir du métal, les produits (I) de la transformation de $\mathrm{Mo}\left(\mathrm{Si}_{1-y} \mathrm{Ge}_{y}\right)_{2}$ restant dans le cément.

Les cémentations ont été réalisées à 1200 et $1300{ }^{\circ} \mathrm{C}$ avec une série d'alliages $\mathrm{Mo}\left(\mathrm{Si}_{1-y} \mathrm{Ge}_{y}\right)_{2}$ tels que : $0,0375<y<0,3$. Ces valeurs ont été choisies de façon à encadrer les variations de composition de la couche superficielle au cours des recuits, dans les pièces cémentées par $\mathrm{Si}_{0,8} \mathrm{Ge}_{0,2}\left(y_{\mathrm{m}}=0,15\right.$ à 0,0375$)$.

Les revêtements obtenus présentent deux couches qui ont respectivement les mêmes caractéristiques que les parties II.1 et II.2 des couches de diffusion (Fig. 9) :

- la couche superficielle (1) d'épaisseur $e_{1}$ présente un fort gradient de concentration en silicium et germanium ;

- la couche interne (2) d'épaisseur $e_{2}$ est formée de $\mathrm{Mo}_{5} \mathrm{Si}_{3}$ pur.

Elles ont une cristallisation basaltique, mais la section des grains est plus faible d'un facteur 4 à 5 dans la couche superficielle. Leur interface est par ailleurs très irrégulier et on remarque des pénétrations très marquées du germanium aux joints de grains de la couche interne de $\mathrm{Mo}_{5} \mathrm{Si}_{3}$ pur.

Les cinétiques de croissance isotherme de $e_{1}$ et $e_{2}$ sont paraboliques. La température et la teneur du cément en germanium $(y)$ ont les effets suivants sur la composition de la couche superficielle et le rapport des épaisseurs $e_{1} / e_{2}$ :

- à chaque température, la teneur maximale en germanium de la couche 1 croît, comme le rapport $e_{1} / e_{2}$, en fonction de $y ; e_{2}$ devient très faible quand la cémentation est réalisée avec l'alliage $\mathrm{Mo}\left(\mathrm{Si}_{0,7} \mathrm{Ge}_{0,3}\right)_{2}$ (Fig. 10);

- pour chaque cément, la teneur maximale en germanium de la couche 1 croît, alors que 


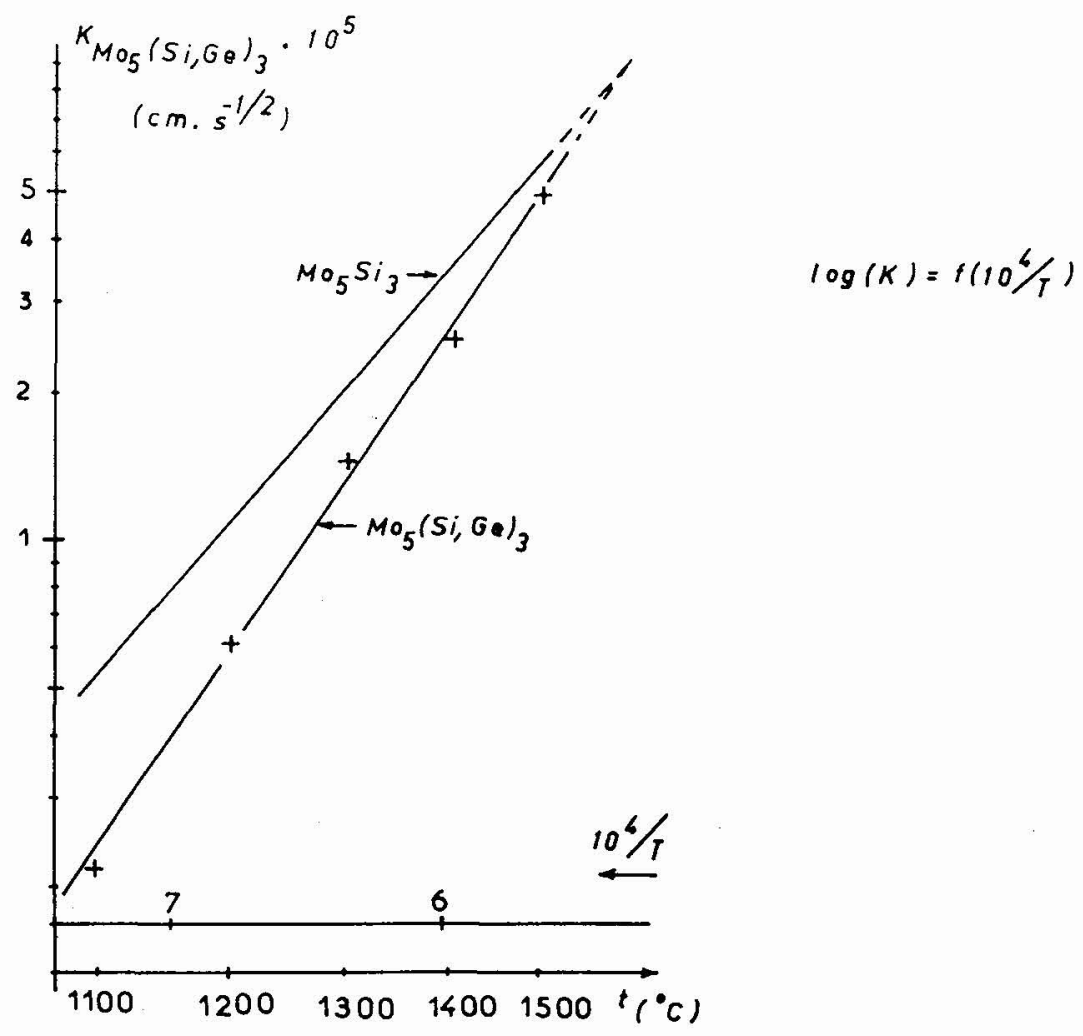

Fig. 8. $-\log \left(K_{\mathrm{Mo5}(\mathrm{Si}, \mathrm{Ge}) 3}\right)=f\left(10^{4} / T\right)$.
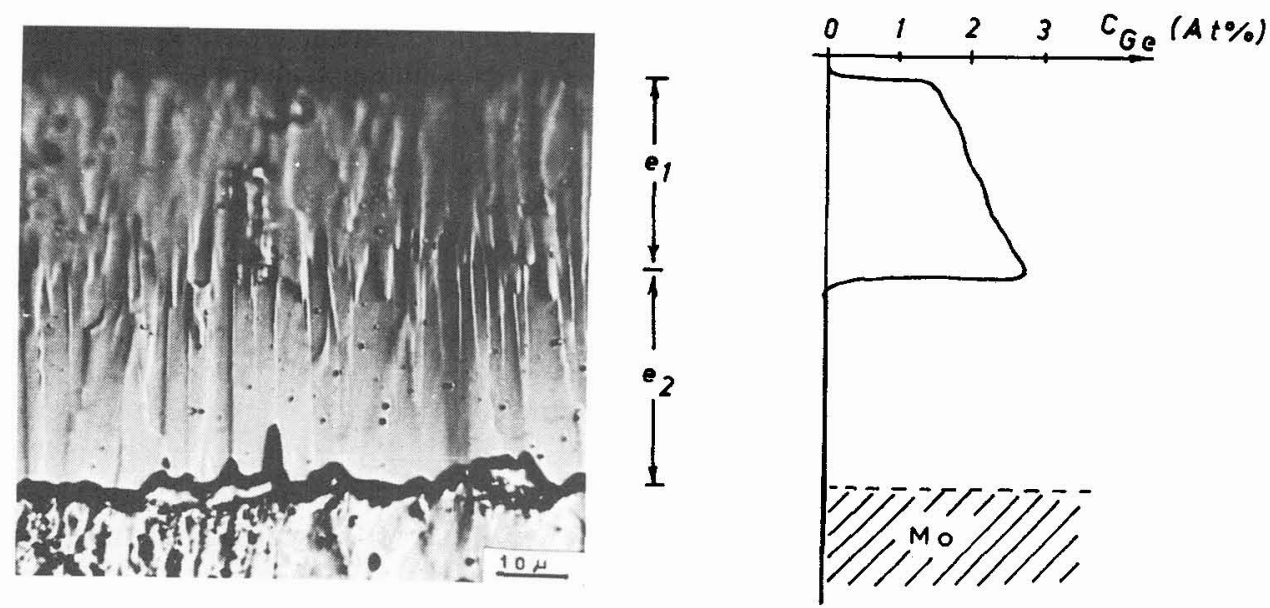

Fig. 9. - Mo cémenté par $\mathrm{MoSi}_{1,925} \mathrm{Ge}_{0,075} 52 \mathrm{~h}$ à $1300^{\circ} \mathrm{C}$. 


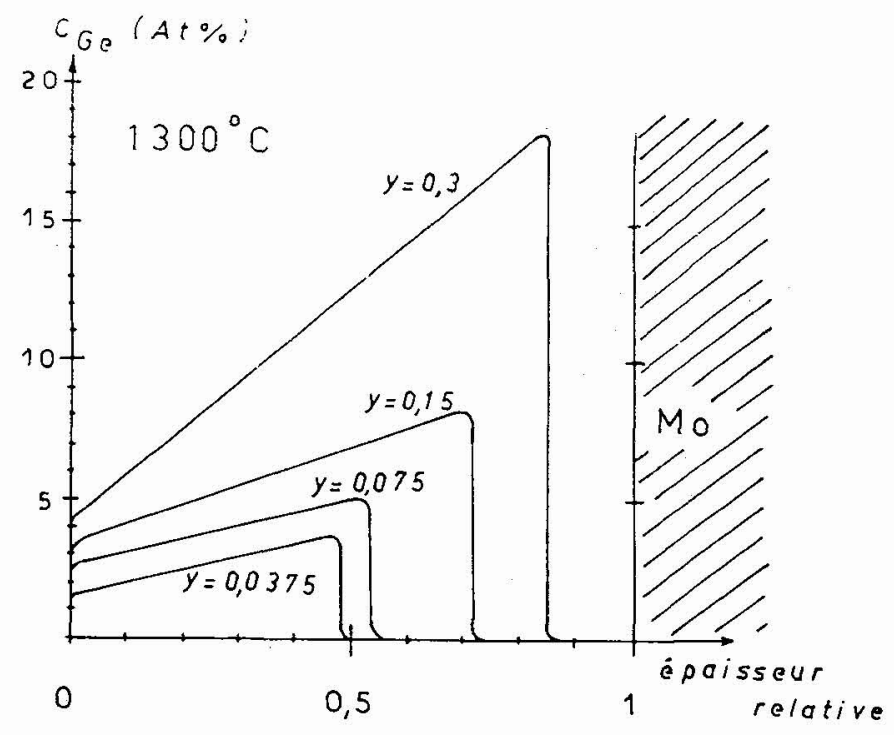

Fig. 10. - Répartition du germanium dans la couche II en fonction de la composition du cément Mo $\left(\mathrm{Si}_{1-y} \mathrm{Ge}_{y}\right)_{2}$.

le rapport $e_{1} / e_{2}$ décroît, en fonction de la température.

On constate en outre des fluctuations de $e_{2}$ dans les pièces traitées assez longtemps par des céments pauvres en germanium, alors que $e_{1}$ est toujours assez régulier (Fig. 11). Aux endroits d'épaisseur $e_{2}$ minimale correspondent un accroissement de la teneur en germanium de la couche superficielle, associé à la formation d'une sous-couche de $\mathrm{Mo}_{3} \mathrm{Si}$ du côté du métal. Le même phénomène est par ailleurs observé lors de la diffusion dans des pièces cémentées par $\mathrm{Si}_{0,8} \mathrm{Ge}_{0,2}$, après des recuits de longue durée (Fig. 5).
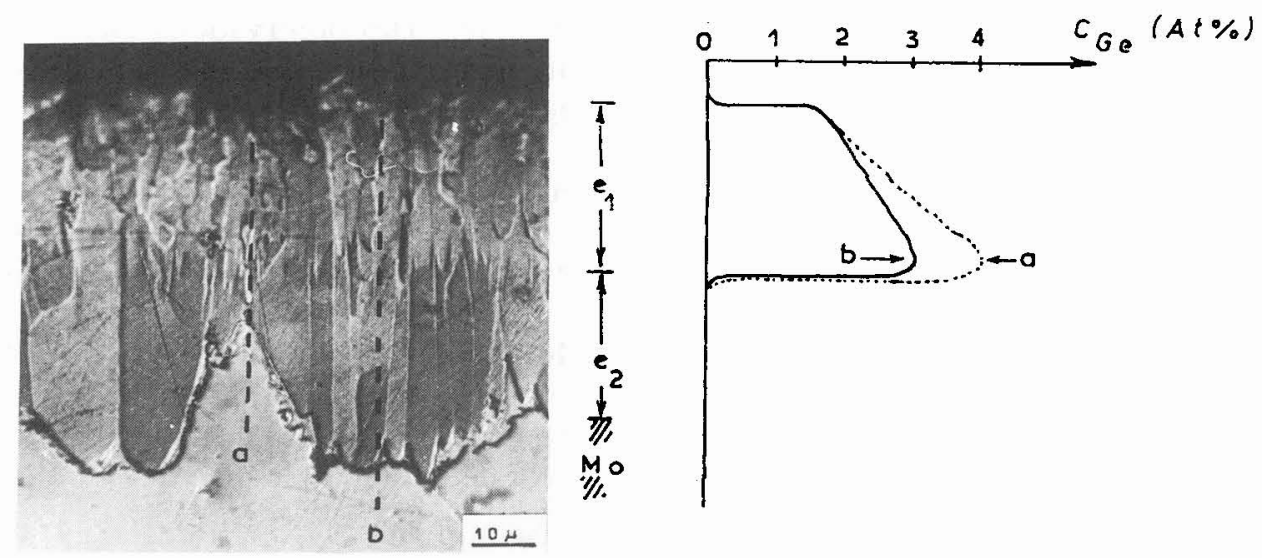

Fig. 11. - Mo cémenté par $\mathrm{MoSi}_{1,925} \mathrm{Ge}_{0,075}$. Distribution du germanium suivant les valeurs de $e_{2}$. 


\section{Discussion.}

Les résultats obtenus concernant la croissance des revêtements de $\mathrm{MoSi}_{2}$ modifiés par le germanium, indiquent que la présence de cet élément n'affecte apparemment pas les mécanismes de diffusion. La diffusion du molybdène dans $\mathrm{MoSi}_{2}$ pur est négligeable, ce qui semble être le cas également en présence de germanium, compte tenu de la morphologie des couches formées, et de la localisation du germanium qui ségrège à l'interface $\mathrm{MoSi}_{2} /$ molybdène. Cette répartition indique par ailleurs que la vitesse de diffusion du germanium dans la structure $\mathrm{C}_{1 \mathrm{lb}}$ est supérieure à celle du silicium.

Le recuit de ces revêtements conduit rapidement à l'homogénéisation de la teneur en germanium, permettant d'assimiler le système métal/revêtement à un couple de diffusion semi-infini $\mathrm{Mo} / \mathrm{Mo}\left(\mathrm{Si}_{1-y} \mathrm{Ge}_{y}\right)_{2}$. On observe ainsi la croissance d'une couche intermédiaire de siliciure de composition proche de celle de $\mathrm{Mo}_{5} \mathrm{Si}_{3}$.

Comme dans le système $\mathrm{Mo} / \mathrm{MoSi}_{2}$, cette couche comporte deux parties. Celle qui se trouve au contact du disiliciure, d'épaisseur $e_{\mathrm{I}}$, est formée par la réaction :

$$
5 \mathrm{Mo}(\mathrm{Si}, \mathrm{Ge})_{2}=>\mathrm{Mo}_{5}(\mathrm{Si}, \mathrm{Ge})_{3}+7(\mathrm{Si}, \mathrm{Ge})
$$

qui se produit à l'interface $\mathrm{Mo}(\mathrm{Si}, \mathrm{Ge})_{2} / \mathrm{Mo}_{5}(\mathrm{Si}, \mathrm{Ge})_{3}$.

Le silicium et le germanium libérés par cette réaction diffusent vers le molybdène, avec lequel ils réagissent suivant la réaction :

$$
7(\mathrm{Si}, \mathrm{Ge})+\frac{35}{3} \mathrm{Mo}=>\frac{7}{3} \mathrm{Mo}_{5}(\mathrm{Si}, \mathrm{Ge})_{3},
$$

qui se produit à l'interface $\mathrm{Mo}_{5}(\mathrm{Si}, \mathrm{Ge})_{3}$. Le rapport $e_{\mathrm{I}} / e_{\mathrm{II}}$ des épaisseurs de $\mathrm{Mo}_{5}(\mathrm{Si}, \mathrm{Ge})_{3}$ formées par les réactions (2) et (3), respectivement, est bien égal à $3 / 7$, comme le prévoit la stoechiométrie de ces réactions. La diffusion du silicium et du germanium est donc prépondérante.

Loriginalité du système ternaire tient à la répartition du germanium dans la couche II. Contrairement au cas de la croissance de $\mathrm{Mo}(\mathrm{Si}, \mathrm{Ge})_{2}$, celle de $\mathrm{Mo}_{5}(\mathrm{Si}, \mathrm{Ge})_{3}$ conduit à une accumulation du germanium dans la partie centrale de la couche de siliciure, comme le confirment par ailleurs les expériences de cémentation du molybdène par $\mathrm{Mo}(\mathrm{Si}, \mathrm{Ge})_{2}$. Il semble donc que le coefficient de diffusion du germanium dans la structure quadratique $\mathrm{D}_{8 \mathrm{~m}}$ de $\mathrm{Mo}_{5} \mathrm{Si}_{3}$, présente une discontinuité importante. Des observations effectuées par ailleurs au microscope électronique sur les échantillons qui viennent d'être décrits, ainsi que sur des couples de diffusion $\mathrm{Mo}_{5} \mathrm{Si}_{3} / \mathrm{Mo}_{5} \mathrm{Ge}_{3}$ [14], indiquent que cette discontinuité s'accompagne d'une diminution importante de la taille de grains de $\mathrm{Mo}_{5} \mathrm{Si}_{3}$, accompagnée d'une ségrégation préférentielle du germanium aux joints.

Linfluence du germanium sur la cinétique de croissance de la couche de $\mathrm{Mo}_{5}(\mathrm{Si}, \mathrm{Ge})_{3}$ est par ailleurs bénéfique, puisqu'elle se traduit par une diminution de la vitesse de croissance de ce composé. A $1100^{\circ} \mathrm{C}$, par exemple, la constante de vitesse est environ deux fois plus faible que celle correspondant à la croissance de $\mathrm{Mo}_{5} \mathrm{Si}_{3}$ dans le système binaire $\mathrm{Mo} / \mathrm{MoSi}_{2}$.

Cet effet cinétique accroît l'intérêt de l'ajout de germanium aux revêtements de $\mathbf{M o S i}_{2}$. Les essais de diffusion montrent en effet que cet élément se répartit très rapidement de façon homogène dans le disiliciure, ce qui devrait être favorable du point de vue de l'oxydation. 


\section{Bibliographie}

[1] FITZer E., Schmidt F.K., High Temp. High Press. 3 (1971) 445.

[2] FitZer E., SChlichting J., Planseeber. Pulvermetall. 20 (1972) 110.

[3] Schlichting J., High Temp. High Press. 10 (3) (1978) 241.

[4] Fitzer E., Remmele W., 5th Int. Conf. on Composite Materials, W.C. Harrigan Jr., J. Strife, A.K. Dhingra Eds. (Met. Soc., 1985) p. 515.

[5] BüCKLE H., 5 Plansee Seminar, Reutte 1964 (Springer, 1965) p. 31.

[6] VerkHorobin L.F., IVANOV V.YE., MATYUSHENKo N.N., NeChiporenKo YE.P., PaGaCHEV N.S., Somov A.I., Fiz. Met. i Metallov. 13 (1962) 77.

[7] IVANOV V.YE., NeChipoRENKo YE.P., ZMIY V.I., Fiz. Met. Metalloved. 17 (1964) 94.

[8] Fitzer E., Matthias K., $6^{\circ}$ Plansee Seminar, Reutte 1968 (Springer, 1969) p. 740.

[9] GaGe P.R., BarTlett R.W., Trans. AIME 233 (1965) 832.

[10] Samsonov G.V., KovaltSchenko M.S., Verkoglja DOVA T.S., Dokl. Akad. Nauk Ukr. RSR 21 (1959) 32.

[11] Bartlett R.W., Gage P.R., LaRSEn P.A., Trans. Met. Soc. AIME 230 (1964) 1528.

[12] Perkins R.A., J. Spacecr. Rockets 2 (1963) 520.

[13] STeinmetz P., Pichoir R., RoQues B., C.R. Acad. Sci. C273 (1971) 309.

[14] STEINMETZ P., Thèse (Nancy, 1977). 